Programmatische Kommentare und Repliken Jakob Tanner*

\title{
Theorieträume der Kulturwissenschaft
}

K) W )

Z ) 
Abstract: My short article is a critical comment on Hartmut Böhme's position paper "Perspectives of cultural studies in historical and contemporary analytical perspective". I share Böhme's conviction that research projects in the broad and blurred interdisciplinary field of culture studies must be grounded in a flexible theoretical plot. However, Böhme's paper does not meet this challenge. I try therefore to suggest two trajectories of significant importance in a paradigmatic way: On the one hand, the always controversial definitions of culture are scrutinized, thereby relying on a context-sensitive concept of "difference" that allows grasping culture as a permanent and interference-prone process of translations. On the other hand, I focus on the impact, agency or effectualness of things against the background of a symmetrical anthropology and the actor-network-theory. In both cases, the analysis is orbiting around the notion of "cultural practices" by demonstrating how tightly the media turn and the ontological turn are theoretically intertwined.

Keywords: Cultural sciences, theory of culture, difference, cultural practices, culture, symmetrical anthropology, actor-network theory, thing theory, material culture, ontological turn, medial turn

*Prof. Dr. Jakob Tanner: Forschungsstelle für Sozial- und Wirtschaftsgeschichte, Universität Zürich, Rämistraße 64, $\mathrm{CH}-8001$ Zürich, email: jtanner@hist.uzh.ch

Über die Frage, was Kultur ist, wird debattiert und gestritten, seit dieser Begriff im 19. Jahrhundert Eingang in die Wissenschaft gefunden hat. Das Spiel der Differenz, aus dem Kultur hervorgeht und in dem sie sich laufend verändert, ließ sich durch forcierten Theorieeinsatz noch nie bändigen. Kultur bleibt ein unterdeterminiertes, ein bewegliches und umkämpftes Konzept. Der programmatische Text von Hartmut Böhme zu den Perspektiven der Kulturwissenschaft folgt einem solchen Verständnis. Er ist von einem robusten Vertrauen in die "Unvermeidlichkeit der Kulturwissenschaft" getragen und ringt gleichzeitig mit deren Positionierung und Institutionalisierung (als "Orientierung", als "Fach" oder als „Feld", vgl. Böhme in diesem Heft) innerhalb des weiten Spektrums der Geisteswissenschaften. Im Endeffekt plädiert der Autor für das vorsätzliche Wollen einer "offenen experimentellen Situation" (vgl. Böhme, ebd.). Das ist erhellend und potenziell produktiv - doch ein "Experimentalfeld" will nicht nur offengehalten, sondern auch mit zielführenden Versuchen bestückt werden, und dazu ist im Text (zu) wenig zu finden...

Der Duktus des Textes ist durchwirkt vom symbolisch hochkarätigen Anlass der Gründung der Kulturwissenschaftlichen Gesellschaft, die auch die vorliegende Zeitschrift herausgibt. ${ }^{1}$ Der

1 Im Folgenden wird laufend auf den in diesem Heft abgedruckten Vortrag von Hartmut Böhme Bezug genommen.
Vortragende verbreitet eine wichtige implizite Botschaft: Ohne breiten Wissensfundus, ohne innovative Denkleistung geht hier gar nichts. Eine kulturwissenschaftliche Forschung auf der Höhe der Zeit wird sich nie mit einigen standardisierten Verfahren der Erkenntnisproduktion begnügen können. Sie muss sich vielmehr im konstitutiv prekären Reflexionsstil der ,Moderne' üben, was wiederum eine Atmosphäre wissenschaftlicher Neugierde und Risikobereitschaft wie bei jeder anderen wissenschaftlichen Disziplin voraussetzt. Die freie Sicht auf ein weites Feld, die Hartmut Böhme mit seinem Bekenntnis zur Offenheit verspricht, wird allerdings durch die massive Präsenz von Bekanntem eingeschränkt. Es entstehen beeindruckende Panoramabilder von Problemlandschaften, die den Blick nicht nur schärfen, sondern auch verstellen können.

Das ist ein performativer Widerspruch, dem wie die Theorie des blinden Flecks nachweist nicht leicht zu entkommen ist. Er wird durch einen weiteren überlagert. Nötig sei, so Böhme, eine "stärkere theoretische Anstrengung" der Kulturwissenschaft. Forschungsfelder und Analyseschwerpunkte bedürfen der "Kulturtheorie(n)" (vgl. Böhme, ebd.). Zurückgewiesen wird ein bloßes "Additionsspiel" oder eine "Summation", die das Ensemble der Kulturwissenschaft zu

Die Anmerkungen sind knapp gehalten. 
einer Resultante von Kraftfeldverlagerungen in Einzeldisziplinen herabstufen würde. Was dann allerdings folgt, ist ein theoretisch nicht weiter begründeter Husarenritt durch vier "große Themen auf engstem Raum!" (vgl. Böhme, ebd.). Der Autor glänzt hier mit einer dichten Präsentation heterogener Forschungsergebnisse, über denen ambitionierte Thesen aufgespannt werden. Die Kaskade großer Namen wird kombiniert mit einer umsichtigen Zitatenlese aus kulturwissenschaftlich kanonisierter Höhenkammliteratur. Solche Belesenheit hat etwas Faszinierendes; nichtsdestotrotz ergibt sich ein Problem, das sich nicht beheben lässt - auch nicht mit der präventiv gemachten Bemerkung, es würden hier "nur exemplarische Felder historischer Forschung vorgestellt" (vgl. Böhme, ebd., Anm.1).

Das themenzentrierte Vorgehen hat zusätzliche Tücken. Zu den vier gewählten Großthemen, welche die letzten 250 Jahre abdecken, sind allein in den letzten Jahren viele Studien publiziert und Debatten geführt worden. Die eigenwillige Bündelung von Forschungsergebnissen, wie sie in Böhmes Text vorgenommen wird, wirkt innerhalb dieses pulsierenden Magmas von Theorietrümmern und Ergebnisbrocken oft etwas abgestanden. Und zu häufig stolpert der geneigte Leser oder die geneigte Leserin über Ungereimtheiten und banale Verkürzungen, etwa beim "halsstarrigen Widerstand des Körpers gegenüber unbegrenztem Fortschritt" oder bei der "Vertigo-Moderne" (vgl. Böhme, ebd.). Adjektive wie "modern", "westlich", "europäisch" und „aufgeklärt" purzeln wild durcheinander und bei Punkt 5 kommt es zu irritierenden semantischen Interferenzen zwischen "Kultur" und "Religion", die weniger der unvermeidlichen theoretischen Unschärfe dieser Begriffe denn ihrem unverbindlichen Einsatz geschuldet sind. Angesichts der eingeforderten, aber nicht eingelösten kulturtheoretischen Fundierung des ganzen Projekts stellt sich bei denjenigen Lesern, welche die Konjunkturen der Kulturwissenschaft(en) im deutschsprachigen Raum seit Mitte der 1980er Jahre verfolgt haben, das Gefühl eines beharrlichen Tretens an Ort und Stelle ein.

$*$

Doch wo müsste die Suche nach einer Kulturwissenschaft, die sich auf der Höhe der Gegenwartsprobleme mit Fragen nach der Zukunft befasst und dabei auch neue Perspektiven auf die Vergangenheit ermöglicht, beginnen? Und in welche Richtung könnte sie gehen? Mit dem zwar weitsichtigen, mit komplexen Fragen ringenden, jedoch auf "anthropologische Konstanten" (vgl. Böhme, ebd.) verbohrten Max Scheler lässt sich heute kaum mehr die Folie aufspannen, auf der die basalen Fragen einer Kulturwissenschaft des 21. Jahrhunderts Konturen annehmen. Die theoretische Unterbelichtung dieses inter- und transdisziplinären Feldes ließe sich eher mit Hinweisen auf angelsächsische und französische Innovationen beheben, wie sie etwa in der "Writing Culture"Debatte (angestoßen durch James Clifford und George M. Marcus 1986) und in der psychoanalytisch inspirierten Reflexion über "Angst und Methode" in den Wahrheitsmaschinen der Wissenschaft (bei Georges Devereux 1976) zu finden wären. Erwähnung verdiente ebenso der seit den 1960er Jahren laufende heiße Theoriebrüter der "Cultural Studies", in dem Semiotik, Marxismus, feministische Theorie, Ethnographie, Poststrukturialismus und -kolonialismus, Literatur- und Medientheorie, Kommunikations- und Kunstgeschichte sowie Intersektionalismus einen nicht abbrechenden theoretischen Energiestrom generierten (besonders wichtig: Hall (1997); für einen Überblick: Grossberg/Nelson/Treichler (1992)).

Natürlich erwartet niemand von einem Vortrag einen kulturwissenschaftlichen tour d'horizon; wenn aber die Signale einer Sensibilisierung für solche Fragestellungen und Zugangsweisen fehlen, kommt auch keine Aufbruchsstimmung auf. Es dominiert eher der Stolz auf das Erreichte. Und es stellen sich unter der Hand Verlustängste ein, wenn Böhme etwa - auf Scheler rekurrierend - die ausdifferenzierte Moderne durch Kontingenzsteigerung und eine "typische Reflexivitätszunahme" charakterisiert, dann aber festgestellt wird, dass sich wahrscheinlich "auch die westlichen Kulturen in eine Mixtur religiöser Energien verwandeln" (vgl. Böhme, ebd.) würden.

Eine Möglichkeit, das Theorieproblem der Kulturwissenschaft zu ,packen', ohne sich diesen einschränkenden Analyseschemata zu unterwerfen, besteht darin, von zentralen Gegenwartsproblemen und -phänomenen auszugehen und diese in ihrer vertrackt-widersprüchlichen Genealogie zu rekonstruieren. Das hat zugleich den Vorteil, dass politische Relevanzkriterien berücksichtigt werden können, ohne in die Falle einer kurz- 
schlüssigen ,Vorgeschichte der Gegenwart' zu tappen. Auch bei einem solchen Vorgehen lässt sich eine gewisse informierte Willkür nicht vermeiden, allein schon deswegen, weil Probleme ja auch von Akteuren erfunden werden, die zunächst bloß eine Antwort haben, die sie jedoch für unverzichtbar halten. Es war ja nicht zuletzt diese antifunktionalistische Erkenntnis, welche die einfachen ,Challenge-Response'-Modelle der traditionellen Sozialgeschichte ausgehebelt und seit den 1980er Jahren einer ,Kulturgeschichte des Sozialen' zum Durchbruch verholfen hat (Chartier 1989). Wenn wir auf jene Herausforderungen fokussieren, die in der globalen Ökonomie der Aufmerksamkeit herausragen, so lassen sich stichwortartig Problemkomplexe identifizieren: der Zusammenhang von Kultur und Migration, die kulturelle Dynamik von Technik und Medien, der Impact von Verwertungsketten und Märkten (im weitesten Sinne) auf das implizite Verständnis von Kultur (und vice versa) und Weiteres mehr. $\mathrm{Zu}$ den beiden erstgenannten Problemstellungen werden im Folgenden einige Überlegungen entwickelt - wobei theoretische Zugänge und konzeptionelle Fragen im Zentrum stehen.

Das Reden über Migration wird heute stark durch ein katastrophisches Metaphernarsenal geprägt. Es werden ,Flüchtlingswellen' beschworen, welche Nationen ,überschwemmen', falls es nicht gelingt, Dämme, Mauern oder Zäune zu errichten. Seit einigen Jahrzehnten ist die Vorstellung auf dem Vormarsch, Kultur lasse sich über basale Grenzziehungen definieren, was es dann nahelegt, ein bedrohtes ,Innen' oder 'Wir' gegen ein bedrohliches ,Außen' oder ,Anderes' zu schützen. Man wähnt sich in einem ,Kulturkampf und verspürt einen ,clash of civilisations'. Das sind Schlagworte, die zum breitenwirksamen Bild von ,Kulturkreisen' gehören. Der Kreis stellt die ideale Demarkationslinie dar. Als geometrische Form entfaltet er genuine Suggestionskraft: Kreisförmig gefasste Kulturen lassen sich auf die Oberfläche des Globus projizieren, es stellt sich die Illusion einer stabilen kulturellen Ordnung ein, die als Topographie von Gefahren zugleich Ängste provoziert. Die politische Rhetorik des ,Kulturkreises' bietet aufgrund ihres maximalen kognitiven Reduktionismus einen Hebel zur vermeintlichen Minimierung von Bedro- hungen. Sie erfüllt jene, die sich mit dem ,Kreis' identifizieren, mit emotionaler Befriedigung. Es ist ,unsere Kultur', die uns zu Subjekten werden lässt, die uns versichert, wer wir sind und was wir nicht wollen. Es entsteht das phantasmagorische Gefühl einer Identität, die in Abgrenzung zu andern ,Kulturkreisen' - in Ablehnung, Abwehr, Kampf und Krieg - gehärtet wird. Der ,Kulturkreis' war und ist eng mit der Geschichte von Ängsten vor einer ,Überfremdung', mit Sozialdarwinismus und rassistischem Denken verwoben. Der kulturelle Differenzgenerator wird in allen diesen Varianten auf großflächige Synchronisation eingestellt; er arbeitet kumulativ und kollektivierend. Beobachtbare Unterschiede fusionieren mit imaginierten Besonderheiten, die Mixtur wird räumlich homologisiert, so dass der Eindruck einer kulturellen Homogenität entsteht, die sich über eine Reihe von territorial verortbaren Wesenszügen fassen lässt. Ein solches Konzept der Kultur ist fundamental essentialistisch, man stellt sich die Menschen immer zuhause vor, nach dem (beliebig variierbaren) xenophoben Motto "Deutschland den Deutschen", wobei schon immer vorhandene kulturelle Differenzen bewusst ausgeblendet werden.

Eine Gegenposition versucht, diese Abwehrreaktionen auf eine multikulturelle Anerkennungspraxis umzupolen. Doch auch in den dominierenden Formen eines Multikulturalismus, der eine relativistische normative Gleichgültigkeit mit einer lebenspraktischen Gleich-Gültigkeit verbindet, steckt ein ähnlicher Kulturessentialismus wie in den Kulturkreis-Theorien. Nur wird hier für ein friedlich-produktives Nebeneinander der Kollektividentitäten plädiert. ${ }^{2}$ Vor allem Migration wird anders, nämlich als Normalzustand wahrgenommen. Während die großräumigen Kulturkreis-Theorien eine starke Zuwanderung als negativ bewerten und diese nur unter bestimmten Anpassungsbedingungen und Assimilationsdruck zulassen möchten, werten multikulturelle Konzepte die Mobilität von Menschen eher positiv und beschreiben deren Resultat im Bild eines kleinräumigen Flickenteppichs, auf dem sich die Kulturen respektieren, d.h. auch tolerieren.

Gegen die mentale Kulturkreis-Verhärtung, die auch in toleranten, multikulturellen Formen noch

2 Die Kritik am Begriff, kulturelle Vielfalt' und am darauf rekurrierenden Multikulturalismuskonzept wurde vor allem durch Homi K. Bhabha formuliert. Siehe Rutherford (1990). 
durchscheint, lässt sich Kultur auch anders, nämlich nicht über eine partikular-homogenisierende Vorstellung von „Vielfalt", sondern über alle holistischen Konzepte subvertierende "Differenz" fassen. Sie erscheint dann als permanente Übersetzungspraxis, durch die Hybridität produziert wird. ${ }^{3}$ Dabei verweist hybrid nicht - wie in der Biologie - auf die Vermischung von zwei "Arten" in einer daraus entstehenden neuen Mischform, sondern auf die Ermöglichungsbedingung von Freiheit: Hybridität schafft vielmehr Aushandlungsorte und Handlungsräume, in denen sich "dritte Räume" öffnen, in denen Menschen frei sein können. Solche permanenten Transkulturations- und Austauschprozesse spielen sich in ganz unterschiedlichen räumlichen und sozialen Konfigurationen ab, die sich weder in einen homogenen Kulturraum noch in einen Kollektivsingular einpassen lassen.

Die Meinung, dass die Durchsetzung der Moderne mit dem Siegeszug eines aufklärerischselbstreflexiven Subjekttypus gleichzusetzen sei, wird fraglich. Menschen sind sich, so die Voraussetzung Homi Bhabhas (1997), vielmehr selber fremd, "wir" sind, so die Formulierung der philosophischen Psychoanalytikerin Julia Kristeva aus den 1980er Jahren, "Etrangers à nous-mêmes" (Kristeva 1988). Die Abwehr eines von außen kommenden Fremden erweist sich aus dieser Sicht als Verdrängung eigener Fremdheitserfahrungen, die auch in Exotisierung und andere Formen eines verklärenden ,Othering' umschlagen kann. Auf der Suche nach Kultur bewegen sich alle Subjekte in einen Zustand hinein, in welchem sie, lost in translation' sind. Die Metapher der Übersetzung wirkt korrosiv auf Kulturessentialismen und stärkt die Sensibilität für kulturelle Grammatiken und Codes. Konzepte wie ,multiple Identitäten', transkulturelle Begehren und globale Netzwerke ermöglichen es, lokale Selbsttechniken und Identifikationsformen auf nationale Resonanzräume, auf religiöse Bindekräfte und kulturellen Wandel im Weltmaßstab zu beziehen. ${ }^{4}$ Werden die Probleme auf diese Weise zurechtgerückt, so wird es z.B. sinnvoll, ,das Religiöse' nicht (wie im Vortrag Böhmes) mit "den Religionen" gleichzusetzen, sondern eine Binde- und Sprengkraft stärker aus

3 Bhabha (1997); vgl. auch: Bronfen/Marius/Steffen (1997); Jäger/Stanitzek (2002); Bachmann-Medick (2006). 4 Wie diese Beziehungen zu fassen sind, bleibt kontrovers. Vgl. u.a. Appiah (2006); Sen (2006); Ehlers/Lezzi (2003); Benhabib (2002). der Figur der radikalen Kontingenzreduktion herzuleiten, die dann auch die Form des Nationalen oder einer hypostasierten Kultur annehmen kann.

$*$

Zu Beginn der 1940er-Jahre schrieb Marc Bloch, die Geschichtswissenschaft habe davon auszugehen, dass sich "der Mensch stark verändert hat, und zwar sowohl mental als auch in den subtilsten Mechanismen seines Körpers" (Bloch 2002: 49). Seit einigen Jahrzehnten hat sich der biomedizinische Zugriff auf den menschlichen Körper intensiviert. Gentechnik, Präimplantationsdiagnostik und Invitro-Fertilisation, chirurgische und medikamentöse Verfahren sowie Weiteres mehr machen die "Techniken des Körpers" (Mauss 1978) zum wirksamen Momentum neuer Subjektivierungsweisen. Zugleich haben wir uns aus der "Gutenberg-Galaxis" (Marshall McLuhan) hinausbewegt. Mit Cyberspace, Big Data und dem Internet der Dinge wachsen vernetzte Computer in neue Größenordnungen und Wirkungsdimensionen hinein, welche gesellschaftliche Interaktionen und menschliche Bedürfnisse sowie Wünsche gleichermaßen affizieren. Und schließlich wirken die akkumulierten Effekte einer auf hohen Material- und Energiedurchsatz angelegten Konsum- und Freizeitgesellschaft nachweislich auf die planetarischen Lebensbedingungen ein. Mit guten Gründen wird von einem anthropogenen Klimawandel und vom Zeitalter des Anthropozäns gesprochen. Im Lichte dieser aktuellen Problemkonstellation kann die Aussage von Hartmut Böhme, "dass menschliche Kulturen nur analysiert werden können in den historischen Verhältnissen zu dem, was nichtmenschlich ist" (vgl. Böhme, ebd.), auf einen Holzweg führen. Dies ist dann der Fall, wenn unter dem "Nichtmenschlichen" "die Dinge, die Tiere und Pflanzen, die Regionen und Landschaften, das Wetter und das Klima" (vgl. Böhme, ebd.) rubriziert werden. Es wäre hier hilfreich gewesen, sich an die kulturwissenschaftliche Provokation Friedrich Kittlers zu erinnern, für den Ontologie "schlussendlich mit Kulturwissenschaft zusammen(fällt)" und "Natur und Mensch derselben geschichtlichen Technik angehören". ${ }^{5}$

5 Kittler (2000: 239 und 244). Kittler referiert hier Heidegger reifizierend. 
Wenn Sigmund Freuds Figur des Menschen als eines, Prothesengottes' und Marshall McLuhans Rede von den ,extensions of man' zur anthropozentrischen Engführung neigen, dann müssen Menschliches und Nichtmenschliches in einer technik- und medientheoretisch fundierten symmetrischen Anthropologie zusammengedacht werden (Latour 1995; vgl auch Tanner 2004). Bruno Latour hat mit der Akteur-Netzwerk-Theorie (ANT) einen produktiven Vorschlag gemacht (Latour 1999), der allerdings deshalb häufig missverstanden wird, weil der subkutane, Sonntagskatholizismus' Latours, der Sermon und Ritual vor Konzept und Argumentation stellt, übersehen wird (vgl. Latour 2010 und die Kritik von Collins 2012, v.a. 417). Wird Wissenschaft als Performance betrachtet, so lässt sich auch Dingen eine Agency zuschreiben. Das bringt Betrieb in die Beschreibung. Und auch in Gesellschaft und Politik kann das Materielle zu einem imaginären Deus ex machina aufsteigen; in Latours "Parlament der Dinge" ereignet sich Erstaunliches. Gegen solche Belebungsversuche lässt sich noch immer die Sichtweise von Keith Thomas $(1971 ; 1983)$ oder aber das Argument von George Devereux anführen, der schrieb: „Der Mensch reagiert auf die Stummheit der Materie mit Panik. Da er ihre Stummheit verleugnen und seine Panik kontrollieren muss, fühlt er sich dazu veranlasst, physikalische Begebenheiten animistisch zu interpretieren und innen, um sie als ,Antworter' erfahren zu können, ,Bedeutungen' zuschreiben, die sie nicht besitzen". ${ }^{6}$

Die „nicht-existente, transzendentale Beredtheit" (Devereux 1976: 57), die ANT-Partisanen an materiellen Phänomenen entdeckt haben, füllt bereits dicke Bücher - wir befinden uns hier mitten in einem theoretischen Wespennest, das man wohl am besten verlässt, aber nicht ohne einige formierende Ideen Latours mit hinauszutragen. Wenn wir das "A" in ANT nicht mit "Akteur" (konnotiert mit "Agency") sondern mit "Aktant" (konnotiert mit „Wirkung" bzw. „Effekt") ausschreiben, dann kann eine Analyse abheben, die dem Menschen auf den Leib rückt, indem sie sich für die Materialität des Körpers interessiert und "den Menschen als durch die Technik mit dem Leben verbunden darstellt", wie George Canguilhem in seinem weg-

6 Devereux (1976), s. Anhang zu Kapitel III: „Das Trauma von der Stummheit der Materie", S. 55-57. weisenden Aufsatz "Maschine und Organismus" (2007) schrieb. Wenn auf diese Weise "Technik als universelles biologisches Phänomen" erkannt und das Mechanische in das Organische "eingeschrieben" wird, ergibt sich ein neuer Zugang zu den Wirkungen von Prothesen, Implantaten, Reproduktionsmedizin, (Xeno)-Transplantation, Neuroenhancing, etc.) (Canguilhem 2007: 206). Aus gesellschaftlicher Perspektive lässt sich mit demselben Erkenntnisinteresse das Konzept einer "anonymen Geschichte" konkretisieren, wie es Sigfried Giedion in den ausgehenden 1940er Jahren vorgeschlagen hatte. Giedion versucht zu erklären, wie „in ihrer Gesamtheit (...) die bescheidenen Dinge", wie aus der mechanisierten Massenproduktion hervorgehen, auf kulturelle Praktiken und den sozialen Wandel einwirken. Er schreibt von "diesen kleinen Dinge des täglichen Lebens", sie würden "sich zu Gewalten (akkumulieren), die jeden erfassen, der sich im Umkreis unserer Zivilisation bewegt." (Giedion 1982: 20)

Weiterführende Anregungen für die kulturwissenschaftliche Erforschung von Dingwelten und Gebrauchsgegenständen lassen sich auch in Vilém Flussers posthum veröffentlichter Essaysammlung Dinge und Undinge (1993) gewinnen. Flusser fokussiert die Aufmerksamkeit auf soziokulturelle Aneignungsprozesse, d.h. er interessiert sich dafür, wie Menschen mit Dingen umgehen, indem sie sich ihrer bedienen, im Wissen darum, dass es "in Wirklichkeit" die Dinge sind, die bedient werden (Flusser 1993: 7). Flusser versteht alle "Dinge meiner Umgebung" unterschiedslos als "meine Bedingung"; er sieht, dass sich Dinge gegen ernsthafte Klassifizierungsversuche sträuben, sortiert sie aber dennoch in drei Gruppen: (1) technische Apparate, die eine "hohe innere Komplexität" haben, über deren Funktionsmodus auch informierte Leute nur eine "sehr verschwommene Kenntnis" haben; (2) Dinge, die er glaubt "verachten zu dürfen", weil sie entweder als "dummes Zeug" nutz- oder funktionslos oder einfach irgendwie kaputt herumstehen oder aber ubiquitär verfügbar sind, so dass man ihnen nichts mehr abgewinnen kann; (3) "Dinge in meiner Umgebung, welche ich schätze", weil sie durch die Investition physischer, wirtschaftlicher, geistiger, emotioneller oder anderer Energien 
teuer geworden sind. Der springende Punkt ist nun der, dass Flusser darüber hinaus eine große Menge von Dingen beobachtet, "die unter keinen der drei genannten Sammelnamen fallen", wozu vor allem jene zählen, "für die sich die Naturwissenschaften interessieren" (Flusser 1993: 7-9).

Der Flussersche Dingsortimenter kann zwei Überlegungen plausibel machen: Erstens ist es nicht sinnvoll, kulturwissenschaftliche Interdisziplinarität auf geisteswissenschaftliche Disziplinen zu beschränken (wie das in Böhmes Text nahegelegt wird). Selbstverständlich gehören die Sozialwissenschaften sowie die Naturwissenschaften, die sciences, mit dazu. Kulturwissenschaft muss die Behauptung der "zwei Kulturen der Wissenschaft" unterlaufen und transversale wissensgeschichtliche Ansätze, die quer zur Aufstellung der Fakultäten liegen, erproben. Und zweitens ist Flussers kognitive und emotionale Ambivalenz ernst zu nehmen. Er betont, es gehe inm mit seiner Dreiteilung darum, "vielleicht etwas zum Verständnis und zur Veränderung unserer Bedingung beizutragen" und gleichzeitig hegt er die Erwartung, „im Gewöhnlichen und Gewohnten Unerwartetes zu entdecken"? (Flusser 1993: 11) Mit dieser Idee eines, Überschusses', der in den Dingen steckt, verbindet sich ein Interesse an "wild things", wie sie in Judy Attfields Studie (2000) über die "material culture of everyday life" genannt werden. ${ }^{7}$ Die Kulturwissenschaft sollte analytisch sensibilisieren für das Überraschungs- und Irritationspotenzial von Dingen, für die Widerständigkeit von Apparaten und für die Eigenlogiken einer massenhaften Inbetriebsetzung industriell fabrizierter Produkte.

Technik verweist auf Medien. Kulturwissenschaftlich bahnbrechend sind die Argumente, die Siegfried Kracauer in seinem 1960 publizierten Buch zum Stummfilm unter dem Titel The Redemption of Physical Reality anstellte. Die deutsche Übersetzung Die Errettung der äußeren Wirklichkeit hat die Meinung bestärkt, Kracauer wolle sagen, dass das Kino Einblicke in die Welt konserviert, die ansonsten sang- und klanglos verschwunden wären. Dabei ging es inm weder um Konserven noch um Rettung. Vielmehr konzipierte er Mentalität und Materialität medientheoretisch neu. Der Witz seiner Filmtheorie besteht darin, dass sie den Einbruch einer bisher nicht gesehenen Wirklichkeit in menschliche

7 Siehe auch: Brewer/Porter (1994); Appadurai (1986).
Erfahrungsräume nicht mit Einstellungswandel, sondern mit Innovationen in Mediendispositiven erklärt. Neue technische Medien lösen jene Wahrnehmungsverschiebungen und Blickverrückungen aus, welche die Stellung und das Selbstverständnis des Menschen in der Welt verändern. Der ,ontological turn' erweist sich so als ,medial turn'. Menschen sehen real existierende Dinge in bestimmter Weise, sie nehmen sie durch Konventionen hindurch wahr, modellieren sie entlang von Deutungsschemata. Verloren geht dabei die Einsicht, dass Dinge auch ganz anders sein und sich auf neue Weise zeigen könnten. Kracauer wollte dem Verstellten, dem Verkannten, dem Unsichtbaren, dem Überraschenden, kurz: dem phänomenalen Reichtum der Welt, zu seinem Recht verhelfen. Das Argument lässt sich zuspitzen: Weil die "äußere Wirklichkeit" in ihren Äußerungsformen unerschöpflich ist, wird sie durch Medien als eine für den Menschen zugängliche überhaupt erst geschaffen. Das filmtheoretische Werk Kracauers lässt den Gegensatz zwischen Realismus und Konstruktivismus hinter sich und kann als produktive Variante einer symmetrischen Anthropologie gelesen werden: Menschen verhelfen mit ihren kommunikativen Praktiken und Interaktionsmustern den Medien auf die Sprünge und letztere verführen wiederum Menschen zu neuen Handlungsweisen, Wahrnehmungen und Selbstverortungen in der Welt.

Solche Problematisierungen, die sich durch die Beobachtung der Gegenwart ergeben, eröffnen neue analytische Zugänge zu großen kulturwissenschaftlichen Themen. Sie rücken relevante Fragen in den Fokus der Forschung. Sie können im Experimentalraum der Kulturwissenschaft, den Hartmut Böhme fordert, neue Denkversuche und Theorietests anregen. Dabei ergeben sich bisher nur wenig explorierte Schnittflächen zu einer "Geschichte des Wissens", die ebenso themenoffen ist und einen ähnlichen Gegenwartsbezug aufweist wie die Kulturwissenschaft.

Grundlegend für kulturwissenschaftliche Praktiken der Reflexion und Erkenntnisgewinnung ist die Einsicht, dass die Auseinandersetzung um den Kulturbegriff integraler Bestandteil von Kultur ist. Es gibt nicht, die Kultur', die man dann post festum noch richtig oder falsch beschreiben und 
erklären könnte. Kultur ist ein reflexives Kraftfeld, das seine Erklärungs- und vielleicht auch Aufklärungsleistungen auf sich selber zurückwendet mit oder ohne Absicht. Keine Definition von Differenz ist unschuldig und in jeder Theorie klingen auch die Träume der Gesellschaft an. Mit George Devereux könnte man ,Gegenübertragungskräfte' am Werk sehen, welche die Kulturwissenschaft im Zuge ihrer Beobachtung der Kultur selber verändern. Dass es aus dieser Dynamik der Beobachtung zweiter Ordnung kein Entkommen gibt, ist eine gleich zu Beginn geäußerte Grundeinsicht von Hartmut Böhme. In der Reflexion auf diese Zirkularität kommen unsere vielfach auseinanderliegenden Positionen zusammen. Kulturwissenschaft ist wichtig - auch politisch!

\section{Literaturverzeichnis}

Appadurai, Arjun (Hg.) (1986): The Social Life of Things: Commodities in Cultural Perspective. Cambridge: Cambridge University Press.

Appiah, Kwame Anthony (2006): The Ethics if Identity. Princeton N.J.: Princeton University Press.

Attfield, Judy (2000): Wild things: the material culture of everyday life. Oxford: Berg.

Bachmann-Medick, Doris (2006): Cultural Turns. Neuorientierungen in den Kulturwissenschaften. Reinbek bei Hamburg: Rowohlt.

Benhabib, Seyla (2002): The Claims of Culture. Equality and Diversity in the Global Era. Princeton N.J.: Princeton University Press.

Bhabha, Homi K. (1997): The location of culture. London: Routledge.

Bloch, Marc (2002): Apologie der Geschichtswissenschaft oder Der Beruf des Historikers (hg. von Peter Schöttler). Stuttgart: Klett-Cotta.

Brewer, John/Porter, Roy (Hgg.): Consumption and the World of Goods, Routledge: London, New York 1994

Bronfen, Elisabeth/Marius, Benjamin/Steffen, Therese (Hgg.) (1997): Hybride Kulturen: Beiträge zur angloamerikanischen Multikulturalismusdebatte. Tübingen: Stauffenburg-Verlag.

Canguilhem, Georges (2007): Maschine und Organismus. In: Nach Feierabend. Zürcher Jahrbuch für Wissensgeschichte 3, S. 185-211.

Chartier, Roger (1989): Kulturgeschichte zwischen Repräsentationen und Praktiken (Einleitung). In: ders. : Die unvollendete Vergangenheit. Geschichte und die Macht der Weltauslegung. Berlin: Klaus Wagenbach, S. 7-19.

Clifford, James/Marcus, George E. (Hgg.) (1986): Writing Culture. The Poetics and Politics of Ethnography. Berkeley: University of California Press.
Collins, Harry (2012): Performances and arguments. Bruno Latour: The modern cult of the factish Gods. In: Metascience 21, S. 409-418.

Devereux, Georges (1976): Angst und Methode in den Verhaltenswissenschaften. Frankfurt a.M. et al.: Ullstein.

Ehlers, Monika/Lezzi, Eva (2003): Fremdes Begehren. Transkulturelle Beziehungen in Literatur, Kunst und Medien. Köln: Böhlau Verlag.

Flusser, Vilém (1993): Dinge und Undinge. Phänomenologische Skizzen. München/Wien: Hanser.

Giedion, Sigfried (1982): Die Herrschaft der Mechanisierung. Ein Beitrag zur anonymen Geschichte (mit einem Nachwort von Stanislaus von Moos). Frankfurt a.M.: Europäische Verlagsanstalt.

Grossberg, Lawrence/Nelson, Car/Treichler, Paula A. (Hgg.) (1992): Cultural Studies. New York/ London: Routledge.

Hall, Stuart (1997): Representation: cultural representation and signifying practices, London: Sage in association with The Open University Press.

Jäger, Ludwig/Stanitzek, Georg (Hgg.) (2002): Transkribieren. Medien, Lektüre. München: Fink.

Kittler, Friedrich (2000): Eine Kulturgeschichte der Kulturwissenschaft. München: Fink.

Kracauer, Siegfried (1960): Theory of Film. The Redemption of Physical Reality. New York: Oxford University Press.

Kristeva, Julia (1988): Etrangers à nous-mêmes. Paris: Fayard.

Latour, Bruno (1995): Wir sind nie modern gewesen. Versuch einer symmetrischen Anthropologie. Berlin: Akademie-Verlag.

Latour, Bruno (1999): On recalling ANT. In: Law, John/ Hassard, John (Hgg.): Actor Network Theory and After. Oxford: Blackwell. S. 15-25.

Latour, Bruno (2010): On the Modern Cult of Factish Gods. Durham, NC: Duke University Press.

Mauss, Marcel (1978): Die Techniken des Körpers. In: ders.: Soziologie und Anthropologie; hg. von Wolf Lepenies/Henning Ritter, Band 2. Frankfurt a.M.: Fischer, S. 197-220.

Rutherford, Jonathan (1990): The Third Space. Interview with Homi Bhabha. In.: ders. (Hg.): Identity: Community, Culture, Difference. London: Lawrence and Wishart, S. 207-211

Sen, Amartya K. (2006): Identity and Violence. The Illusion of Destiny. New York: W.W. Norton.

Tanner, Jakob (2004): Historische Anthropologie zur Einführung. Hamburg: Junius.

Thomas, Keith (1971): Religion and the Decline of Magic. Studies in Popular Beliefs in Sixteenth and Seventeenth Century England. London: Weidenfeld/ Nicolson.

Thomas, Keith (1983): Man and the Natural World: A History of Modern Sensibility, New York: Pantheon Books. 